\title{
CONHECIMENTOS SOBRE SAÚDE OCULAR ENTRE PROFISSIONAIS DE UM HOSPITAL UNIVERSITÁRIO
}

\author{
KNOWLEDGE ON OCULAR HEALTH AMONG UNIVERSITY HOSPITAL STAFF
}

Edméa Rita Temporini' ${ }^{1}$ N Newton Kara-José1; Éverton Lima Gondim² \& Fernando Justino Dantas ${ }^{3}$

\begin{abstract}
${ }^{1}$ Docentes Disciplina de Oftalmologia da Faculdade de Medicina -USP e da Faculdade de Ciências Médicas - UNICAMP. ${ }^{2}$ Pós-graduando na Disciplina de Oftalmologia da FCM-UNICAMP. ${ }^{4}$ Residente de Oftalmologia FCM-UNICAMP.

CoRRESPONDÊNCIA: Prof.Newton Kara-JosConhecimentos sobre saúde ocular entre profissionais de um hospital universitário. Divisão de Clínica Oftalmológica do Hospital das Clínicas da FMUSP. Av. Dr. Enéas de Carvalho Aguiar, 255 - $6^{\circ}$ andar - sala 6121 Fax: (011) 38162287 - CEP: 05403-000 - São Paulo-S.P.
\end{abstract}

TEMPORINI ER; KARA-JOSÉ N; GONDIM EL \& DANTAS FJ. Conhecimentos sobre saúde ocular entre profissionais de um hospital universitário. Medicina, Ribeirão Preto, 35: 53-61, jan/mar.2002.

RESUMO: Observacional exploratório. OBJETIVO: Verificar conhecimentos sobre saúde ocular entre profissionais de diferentes categorias e áreas de especialidade em um hospital universitário. MÉTODOS: Aplicou-se questionário não-estruturado sobre afecções oculares (catarata, glaucoma e retinopatia diabética) e conduta em queimadura química ocular. RESULTADOS: A amostra foi formada por 122 sujeitos: 30 enfermeiros (24,6\%), 52 auxiliares de enfermagem (42,6\%), 24 auxiliares administrativos $(19,7 \%)$ e 16 funcionários do serviço de oftalmologia $(13,1 \%)$ que se dispuseram a participar da pesquisa. Afirmaram saber o que é catarata $83,3 \%$ dos enfermeiros e $75,0 \%$ do pessoal de oftalmologia. Entre os grupos não atuantes em oftalmologia observou-se definição incorreta dessa afecção. Resumo - Acrescentar: " O glaucoma é desconhecido por $79,1 \%$ dos funcionários administrativos do hospital; $37,6 \%$ do pessoal do serviço de oftalmologia declaram não saber a respeito de cura ou controle dessa afecção. O desconhecimento acerca das conseqüências do diabetes para o sistema visual foi observado em todos os grupos. CONCLUSÃO: O desconhecimento a respeito das afecções oculares ocorreu em todas as categorias. Sugere-se o desenvolvimento de programas de treinamento de funcionários hospitalares, visando à multiplicação de orientações à clientela e no meio sóciocultural.

UNITERMOS: Saúde Ocular. Educação em Saúde. Equipe de Assistência ao Paciente. Cegueira; prevenção.

\section{INTRODUÇÃO}

O conhecimento em relação à saúde ocular constitui condição necessária e antecedente às ações do indivíduo para preservar a visão. Embora reconheça-se que a ampliação e a melhoria do conhecimento "per se" nem sempre resultem em mudança comportamental, conjuntamente às atitudes, crenças, valores e percepções do indivíduo, compõem o grupo de fato- res responsáveis pela justificativa ou motivação para recusar ou adotar um comportamento específico. Esses fatores incluem dimensões cognitivas e afetivas do saber, sentir, acreditar, valorizar e da autoconfiança ou percepção da própria competência para agir de determinada maneira ${ }^{(1)}$.

Tais aspectos devem merecer especial atenção na medida em que se pretenda obter um diagnóstico prévio da realidade, que antecede alguma interven- 
ção. Desse modo, pode-se direcionar apropriadamente o planejamento de ações de saúde, tendo em vista grupos-alvo diverso ${ }^{(2)}$.

Constitui propósito da educação em saúde, além da transmissão de informações, a transformação de conhecimentos, atitudes e condutas existentes, visando à promoção e preservação da saúde ${ }^{(3)}$. As ações educativas, portanto, constroem a base para a promoção da saúde ocular e preservação do sistema visual, aumentando a capacidade dos indivíduos de tomar decisões relativas a comportamentos que influenciarão seu nível de saúde ocular.

De acordo com Green \& $\operatorname{Kreuter}^{(1)}$, educação em saúde consiste em qualquer combinação de experiências aprendidas para facilitar ações voluntárias que conduzam à saúde. As ações de educação em saúde, portanto, apresentam a finalidade principal de aumentar a capacidade dos indivíduos de tomar decisões e agir corretamente em relação a assuntos de saúde, processo que inclui dimensões intelectuais, psicológicas e sociais ${ }^{(3)}$.

A educação em saúde é de responsabilidade de todos que mantem contato com a população usuária de serviços de saúde, nas unidades de atendimento: médicos, enfermeiros, auxiliares e demais profissionais. Os objetivos de programas e ações de educação em saúde, portanto, devem incluir a obtenção ou aprofundamento de conhecimentos, atitudes e práticas do pessoal especialista, técnico e auxiliar, além da cooperação dos indivíduos envolvidos ${ }^{(4)}$.

Os profissionais atuantes em ambiente hospitalar, particularmente em serviço de oftalmologia, devem deter conhecimentos científicos essenciais a respeito de afecções oculares. Em razão da natureza de suas funções, prestam informações sobre saúde à clientela ou atuam como multiplicadores de orientação e, por vezes, provêm esclarecimentos a pessoas pertencentes ao seu meio social. Por outro lado, a maior parte das doenças oculares evitáveis requer ações multidisciplinares de que participam oftalmologistas, epidemiologistas, educadores de saúde, enfermeiros e outros especialistas, além do pessoal auxiliar ${ }^{(5)}$.

Vale ressaltar a importância de difundir informações sobre saúde ocular à população, com vistas à realização de ações adequadas, pois do reconhecimento da presença de afecção ocular e da conduta apropriada do paciente depende, em grande parte, o sucesso do prognóstico oftalmológico.

$\mathrm{O}$ ensino formal e as atividades de educação continuada, ao lado das experiências de vida, concor- rem para a construção do conhecimento que esses profissionais devem ter, formado a partir de duas vertentes: a representada pelo saber científico e a que tem origem no meio sociocultural do qual fazem parte.

Torna-se necessário, portanto, o preparo do pessoal de serviço hospitalar, com ênfase no serviço de oftalmologia, no sentido de alertá-lo sobre as necessidades da clientela em relação à saúde ocular e à importância da maneira apropriada de prover esse atendimento. Assim, essa preparação deve incluir atividades e informações que eliminem conhecimentos errôneos e mitos, bem como introduzir metodologias de educação participativa que contemplem as diversidades socioculturais ${ }^{(6,7)}$.

Esse esforço preventivo é amplamente justificado uma vez que a orientação correta configura-se em medida de prevenção de cegueira e de transtornos visuais. Ressalta-se que a cegueira e a incapacidade visual acarretam conseqüências sociais, psicológicas e econômicas adversas para o indivíduo e para a sociedade ${ }^{(5)}$.

A indagação principal do presente estudo originou-se da busca da comprovação da lacuna existente acerca do conhecimento de profissionais atuantes em ambiente hospitalar sobre saúde ocular. Foram investigados conhecimentos básicos sobre determinadas afecções oculares entre profissionais dos níveis técnico, auxiliar e administrativo, atuantes em hospital universitário, a fim de obter informações para subsidiar intervenções educativas de preparação de pessoal em saúde ocular.

\section{MATERIAL E MÉTODOS}

Foi realizada uma pesquisa exploratória, focalizando conhecimentos referentes a afecções oculares entre profissionais de hospital. Escolheu-se esse método de pesquisa por se tratar de tema sobre o qual a literatura oferecia raros subsídios, ao mesmo tempo em que se reconhecia a importância de identificar possíveis falhas e lacunas de conhecimento entre pessoal atuante na área de saúde, visando ao planejamento de intervenções educativas. Foi desenvolvida uma investigação inicial para adquirir melhor compreensão dessa realidade.

A amostra foi constituída por pessoal de enfermagem (de nível superior e auxiliar) e por funcionários administrativos, atuantes em diferentes clínicas e setores do Hospital das Clínicas da Universidade de Campinas (Brasil), que se dispuseram a participar do 
estudo. Por se tratar de amostra intencional, não é possível fazer generalizações.

Foi utilizado um questionário auto-aplicável, não estruturado, contendo apenas perguntas a respeito de algumas afecções oculares (catarata, glaucoma e retinopatia diabética) e conduta a ser adotada, no domicílio, em trauma químico, ocular. Esses assuntos foram selecionados em razão da freqüência com que se apresentam na prática médica e por constituírem indicadores válidos do conhecimento em saúde ocular.

Para a coleta de dados, foram fornecidas orientações aos respondentes em relação aos objetivos da pesquisa, anonimato e sigilo das informações prestadas. As respostas foram fornecidas individualmente; os questionários completados foram devolvidos à equipe de pesquisa, que aguardava o seu preenchimento em local próximo, sem interferir nas formas das respostas ou no tempo dispendido pelo respondente, assegurada a ausência de comunicação.

Para a análise dos dados, os sujeitos foram agrupados em categorias, de acordo com a função exercida e o fato de atuarem no serviço de oftalmologia ou em outros serviços hospitalares. Assim, foram estabelecidos os seguintes grupos: enfermeiros, auxiliares de enfermagem e funcionários administrativos, do serviço de oftalmologia e dos demais serviços.

Por tratar-se de questões abertas, as respostas foram categorizadas sem o uso de software, em etapa anterior a do processamento dos dados pelo programa EPI-INFO 6.0.

\section{RESULTADOS}

A amostra foi composta por 122 sujeitos: 30 enfermeiros $(24,6 \%), 52$ auxiliares de enfermagem (42,6\%), 24 funcionários administrativos (19,7\%), atuantes em setores hospitalares diversos, e por 16 funcionários do serviço de oftalmologia (13,1\%).

A maior proporção dos sujeitos declara saber o que é catarata, destacando-se os grupos dos enfermeiros $(83,3 \%)$ e dos funcionários do serviço de oftalmologia $(75,0 \%)$. Entre os que afirmam deter esse conhecimento, nos grupos de profissionais não atuantes em oftalmologia, expressivas proporções $(48,0 \%$, $59,1 \%$ e $92,2 \%$ ) referem-se à catarata como "membrana ou pele cobrindo o olho". (Tabela I)

Em relação ao tratamento da catarata, predomina, acentuadamente, o reconhecimento da existência de tratamento para essa afecção. Contudo, 12,5\% dos funcionários do serviço de oftalmologia declaram ignorar esse fato. A cirurgia é admitida como tratamento preferencial por quase a totalidade dos sujeitos, que reconhecem ser a catarata passível de tratamento. O uso de colírios como forma de tratar a catarata é mencionado por $13,3 \%$ dos enfermeiros. (Tabela II)

Declaram saber o que é glaucoma $56,2 \%$ do pessoal de oftalmologia, porém essa afecção é desconhecida por $23,3 \%$ dos enfermeiros, $61,5 \%$ dos auxiliares de enfermagem e 79,1\% dos funcionários administrativos dos demais serviços pesquisados. Entre os que afirmam conhecer, a possibilidade de cura dessa

\begin{tabular}{|c|c|c|c|c|}
\hline Conhecimento & $\begin{array}{c}\text { Servico de Oftalmoloqia } \\
\text { Funcionários } \\
(\mathrm{r}=16) \\
\%\end{array}$ & $\begin{array}{c}\text { Enfermeiras } \\
\qquad(\mathrm{r}=30) \\
\%\end{array}$ & $\begin{array}{l}\text { Outros servicos } \\
\text { Auxiliares de } \\
\text { Enfermagem } \\
\text { (r= } 52 \text { ) } \\
\%\end{array}$ & $\begin{array}{c}\text { Funcionários } \\
\text { Administrativos } \\
\begin{array}{c}(\mathrm{n}=24) \\
\%\end{array}\end{array}$ \\
\hline \multicolumn{5}{|l|}{ Conhecimento da doenca } \\
\hline Declara conhecer & 75,0 & 83,3 & 59,6 & 54,2 \\
\hline Declara nẫo conhecer & 25,0 & 16,7 & 40,4 & 45,8 \\
\hline Definiçôes de c atarata & $(\mathrm{r}=12)$ & $(\mathrm{r}=25)$ & $(\mathrm{r}=31)$ & $(n=13)$ \\
\hline Opacidade do cristalino & 81.2 & 40,0 & 19,3 & 7.8 \\
\hline Membrana ou pele cobrindo o olho & 12.5 & 48,0 & 59.1 & 92,2 \\
\hline Membrana sobre a retina & - & 8,0 & 11,9 & - \\
\hline Outras & 6.3 & 4,0 & 9.7 & - \\
\hline
\end{tabular}


afecção é aceita por $21,7 \%$ dos enfermeiros, $35,0 \%$ dos auxiliares de enfermagem e por $31,2 \%$ dos funcionários do serviço de oftalmologia. Verificou-se existir desconhecimento do assunto em todas as categorias profissionais, mais acentuadamente entre os funcionários administrativos (79,1\%); 37,6\% do pessoal do serviço de oftalmologia declaram não saber a respeito de cura ou controle do glaucoma. (Tabela III).

A relação entre diabetes e doença ocular é reconhecida por quase a totalidade do pessoal de enfermagem $(100,0 \%$ e $86,5 \%)$ e do serviço de oftalmologia $(87,5 \%)$; a maioria dos respondentes de todas as categorias profissionais declara que os problemas oculares de pacientes diabéticos decorrem do controle inadequado dessa doença. O desconhecimento a respeito das conseqüencias do diabetes para o sistema visual, contudo, está presente em todos os grupos observados. (Tabela IV).

Os dados da Tabela V mostram o conhecimento sobre a forma de proceder, se houver contato de produto químico com os olhos. Cerca de $50,0 \%$ dos funcionários, em todos os grupos, aparentemente conhecem a conduta correta, citando que, antes de procurar atendimento especializado, é necessário lavar os olhos com água corrente. Entre o pessoal do serviço de oftalmologia, 46,7\% mencionam apenas a lavagem com água e $20,0 \%$, a lavagem dos olhos com soro fisiológico.

\begin{tabular}{|c|c|c|c|c|}
\hline Conhecimento sobre c atarata & $\begin{array}{c}\text { Servico de Oftalmologia } \\
\text { Funcionários } \\
(\mathrm{n}=16) \\
\%\end{array}$ & $\begin{array}{c}\text { Enfermeiras } \\
\qquad(\mathrm{n}=30) \\
\%\end{array}$ & $\begin{array}{l}\text { Outros servicos } \\
\text { Auxiliares de } \\
\text { Enfermagem } \\
(\mathrm{r}=52) \\
\%\end{array}$ & $\begin{array}{c}\text { Funcionários } \\
\text { Administrativos } \\
\begin{array}{c}(n=24) \\
\%\end{array}\end{array}$ \\
\hline \multicolumn{5}{|l|}{ Existência de tratamento } \\
\hline Sim & 87,5 & 100,0 & 98,0 & 91,7 \\
\hline Nẫo & - & - & - & - \\
\hline Nẫo sabe & 12,5 & - & 2,0 & 8.3 \\
\hline Tipo de tratamento & $(n=14)$ & $(n=30)$ & $(r=51)$ & $(n=22)$ \\
\hline Ciruraia & 92,9 & 80,0 & 96.0 & 77.3 \\
\hline Colírios & - & 13,3 & 2,0 & - \\
\hline Nẫo sabe & 7.1 & 6.7 & 2,0 & 22,7 \\
\hline
\end{tabular}

\begin{tabular}{|c|c|c|c|c|}
\hline $\begin{array}{l}\text { Combecimento } \\
\text { sobre o diacoma }\end{array}$ & $\begin{array}{c}\text { Servico de Oftalmolodia } \\
\text { Funcionários } \\
(n=16) \\
\%\end{array}$ & $\begin{array}{c}\text { Enfermeiras } \\
\left(\begin{array}{c}n=30) \\
\%\end{array}\right.\end{array}$ & $\begin{array}{c}\text { Outros semicos } \\
\text { Auxiliares de } \\
\text { Enfermagem } \\
(n=52) \\
\%\end{array}$ & $\begin{array}{c}\text { Funcionários } \\
\begin{array}{c}\text { Administrativos } \\
(n=24) \\
\%\end{array}\end{array}$ \\
\hline Declara saber & 56,2 & 76,7 & 38,5 & 20,9 \\
\hline Declara nẫo saber & 43,8 & 23,3 & 61,5 & 79,1 \\
\hline Possibilidade de cura & $(n=9)$ & $(n=23)$ & $(n=20)$ & $(n=5)$ \\
\hline Tem cura & 31,2 & 21,7 & 35,0 & - \\
\hline Não tem cura, apenas controle & 31,2 & 60,9 & 30,0 & 20,0 \\
\hline Nẫo sabe & 37,6 & 17,4 & 35,0 & 80,0 \\
\hline
\end{tabular}




\begin{tabular}{|c|c|c|c|c|}
\hline Relação diabetes / doença ocular & $\begin{array}{c}\text { Serviço de Oftaimalogia } \\
\text { Funcionários } \\
(n=16) \\
\%\end{array}$ & $\begin{array}{c}\text { Enfermeiras } \\
(n=30) \\
\%\end{array}$ & $\begin{array}{c}\text { Outros servicas } \\
\text { Auxillares de } \\
\text { Enfermagem } \\
(r=52) \\
\%\end{array}$ & $\begin{array}{c}\text { Funcionários } \\
\text { Administrativos } \\
\begin{array}{c}(n=24) \\
\%\end{array}\end{array}$ \\
\hline \multicolumn{5}{|l|}{$\begin{array}{l}\text { Relação entre diabetes e doença } \\
\text { ocular }\end{array}$} \\
\hline Tem relaçẫo & 87,5 & 100,0 & 86,5 & 58,3 \\
\hline Nẫo tem relaçẫo & - & - & - & - \\
\hline Não sabe & 12,5 & - & 13,5 & 41,7 \\
\hline Consequiência * & $(n=14)$ & $(n=30)$ & $(n=45)$ & $(n=14)$ \\
\hline Retinopatia & 35,7 & 20,0 & 8,9 & - \\
\hline Cegueira & 7,1 & 13,3 & 35,5 & 4,2 \\
\hline Catarata & 7,1 & - & - & - \\
\hline Hemorragia intra-ocular & 12,3 & - & 2,2 & - \\
\hline Glaucoma & 7,1 & - & - & - \\
\hline Outros & 7,1 & 6,7 & 4,4 & 4,2 \\
\hline Nã̃o sabe & 57,1 & 60,0 & 49,0 & 91,6 \\
\hline
\end{tabular}

\begin{tabular}{|c|c|c|c|c|}
\hline Conhecimento & $\begin{array}{c}\text { Serviço de Oftaimologia } \\
\text { Funcionários } \\
(n=16) \\
\%\end{array}$ & $\begin{array}{c}\text { Enfermeiras } \\
\qquad \begin{array}{c}(n=30) \\
\%\end{array}\end{array}$ & $\begin{array}{c}\text { Outras servicos } \\
\text { Auxiliares de } \\
\text { Enfermagem } \\
(n=52) \\
\%\end{array}$ & $\begin{array}{c}\text { Funcionánios } \\
\text { Aciministrativos } \\
(n=24) \\
\%\end{array}$ \\
\hline Deve-se fazer algo & 93,8 & 93,3 & 94,2 & 83,3 \\
\hline Nẫo há o que ser feito & - & - & 2,0 & - \\
\hline Nã̃o sabe & 6,2 & 6,7 & 3,8 & 16,7 \\
\hline Forma de proceder & $(n=15)$ & $(n=28)$ & $(n=49)$ & $(n=20)$ \\
\hline Lavar com água e procurar & 13,3 & 46,4 & 55,1 & 45,0 \\
\hline \multicolumn{5}{|l|}{ Orientaçẫo médica } \\
\hline Lavar com água & 46,7 & 39,4 & 38,8 & 45,0 \\
\hline Lavar com soro fisiológico & 20,0 & 7,1 & 2,0 & - \\
\hline Outros & 20,0 & 7,1 & 4,1 & 10,0 \\
\hline
\end{tabular}




\section{DISCUSSÃO}

Os conhecimentos errôneos e a ausência de conhecimentos em saúde ocular constituem obstáculos importantes às ações preventivas de agravos e de preservação do sistema visual, realizadas por pessoal da área de saúde junto à comunidade. Esses profissionais devem deter preparo científico compatível às funções de orientação e esclarecimento da clientela hospitalar, precisam ser sensibilizados em relação às necessidades em saúde ocular dos usuários e ao seu atendimento apropriado ${ }^{(6)}$

A realização de programas de treinamento de pessoal de saúde alcança maior eficácia, entretanto, se for obtido conhecimento prévio a respeito de concepções e conduta referentes à saúde ocular. Esse conhecimento permite direcionar a intervenção educativa e evitar insucessos ou utilização inadequada de recursos $^{(2)}$.

As afecções oculares, abordadas neste estudo, incluem-se entre as principais causas de cegueira na América Latina, apontadas por especialistas, em projeção para a oftalmologia do ano $2004^{(8)}$.

A cegueira conseqüente à catarata revela-se problema de saúde pública de proporção considerável nos países em desenvolvimento. Apesar do número crescente de cirurgias de catarata, realizadas em todo o mundo, devido à grande incidência, o número total de cegos por catarata continua aumentando. A Organização Mundial de Saúde (OMS) aponta a catarata como a principal causa de cegueira que atinge de 27 a 35 milhões de pessoas no mundo ${ }^{(9)}$.

Trata-se, contudo, de afecção ocular curável por meio do procedimento cirúrgico de remoção da lente afetada, com prognóstico favorável à restauração da visão. Muitos indivíduos, contudo, permanecem cegos por falta de acesso ou medo da cirurgia $^{(10,11)}$. Embora reconheça-se a gravidade da perda da capacidade visual, o maior obstáculo para o tratamento parece residir no acesso do paciente aos serviços, ao invés da ausência de tecnologia adequada ${ }^{(12,13)}$.

Há necessidade, portanto, de orientar as pessoas de modo a poderem decidir sobre a necessidade de se submeterem à cirurgia, contrapondo o efeito adverso da catarata na sua vida ao risco, embora reduzido, de complicações do ato cirúrgico ${ }^{(9)}$.

Compete ao pessoal de enfermagem complementar a orientação fornecida por oftalmologista e auxiliar a esclarecer o paciente, tarefa que requer conhecimentos corretos a respeito de catarata.
Os resultados observados evidenciam que, embora a maior proporção dos funcionários, em todos os grupos, declarem saber o que é catarata, nota-se que a definição errônea dessa afecção, é manifestada mesmo por pessoal do serviço de oftalmologia (Tabela I). A menção de colírios e o desconhecimento do tipo de tratamento da catarata (Tabela II), fazem supor que esses funcionários não se preocupam em aprofundar conhecimentos sobre essa afecção, contentando-se em dispor apenas de conhecimento superficial. É provável que esses indivíduos, em razão do seu desconhecimento, produzam orientação imperfeita para pacientes e familiares deles e em seu próprio meio social.

Em relação ao glaucoma, acentua-se a proporção dos que declaram seu desconhecimento, em comparação à catarata (Tabela III). Entre os que declaram saber, observa-se também a presença de conhecimento errôneo ou desconhecimento acerca da possibilidade de cura. O glaucoma situa-se entre as principais causas de cegueira em população idosa.

Dados e projeções de 1993, de nível mundial, revelaram que o glaucoma representa problema maior do que se supunha. As intervenções de prevenção da cegueira por glaucoma apresentam muitas dificuldades, em especial nos países em desenvolvimento, onde a detecção precoce e o controle da doença constituem problemas de solução difícil ${ }^{(14,15)}$. Ademais, espera-se, no futuro, a ocorrência do aumento do glaucoma no mundo todo, como reflexo do envelhecimento populacional e da insuficiência de recursos oftalmológicos para intervenção efetiva contra essa afecção ${ }^{(13)}$.

O diagnóstico e tratamento precoces do glaucoma são fundamentais para prevenção da perda visual, porém dependem do conhecimento e da conduta das pessoas, para que se realizem. É de grande importância que funcionários de instituições hospitalares estejam capacitados a orientar e informar pacientes e pessoas do seu meio social a respeito do glaucoma e de sua prevenção. Essa intervenção educativa poderá propiciar a conduta preventiva, necessária em relação ao glaucoma.

O conhecimento acerca da influência do diabetes no sistema visual está presente nos funcionários de áreas técnicas e, de forma menos acentuada, entre o pessoal administrativo. Entretanto, mostra-se expressivo o desconhecimento a respeito das conseqüências do diabetes no sistema ocular (Tabela IV), indicando que, mesmo quando existe conhecimento, ele é superficial. 
A retinopatia diabética representa causa freqüente de cegueira legal em indivíduos de 20 a 74 anos, em nações industrializadas. Especialistas estimam a existência de diabetes não diagnosticado em proporção considerável da população, que permanece sem tratamento por longo tempo ${ }^{(16)}$. Embora não exista cura para a retinopatia diabética, o exame oftalmológico e o tratamento apropriado podem reduzir o risco de perda visual. A busca precoce desse atendimento, não obstante, depende de o indivíduo estar informado a respeito da doença e das complicações oculares decorrentes. É surpreendente a insuficiência de conhecimentos, observada entre o pessoal de enfermagem, neste estudo, considerando-se a importância da sua atuação na equipe de saúde para a prevenção e tratamento de complicações oculares do diabetes (Tabela IV).

Em relação ao conhecimento sobre a conduta em caso de acidente ocular, produzido por contato com produto químico, uma proporção expressiva dos respondentes considera ser necessário lavar os olhos em água corrente, antes de procurar auxílio especializado (Tabela V), o que corresponde ao conhecimento correto.

Pode-se supor que tal concepção seja muito mais intuitiva que devida a conhecimento científico, adquirido. A opção de lavagem com soro físiológico, presente nas respostas do pessoal da área de oftal- mologia, parece indicar a influência do ambiente de trabalho, esquecendo que, no domicílio, deve-se lançar mão do que estiver mais acessível, no caso, a água corrente.

\section{CONCLUSÃO}

Os resultados deste estudo sugerem haver insuficiência de conhecimentos básicos, referentes a todas as afecções oculares focalizadas, por parte de profissionais atuantes em ambiente hospitalar, seja na clínica oftalmológica ou em outros setores.

Concepções falsas ou distorcidas, entre profissionais que atuam em hospital, a respeito de assuntos de saúde ocular mostram a importância de haver prioridade no desenvolvimento de programas de capacitação e treinamento em serviço, visando à multiplicação das orientações à clientela e no meio sociocultural. Somente pessoas informadas podem adotar e orientar comportamento preventivo, desejável para preservar o sistema visual.

\section{Agradecimentos}

Os autores agradecem a Fabrício Teno Castilho Braga e Jorge Carlos Pessoa Rocha, residentes de oftalmologia da Universidade Estadual de Campinas, por sua valiosa participação na coleta e análise dos dados da presente pesquisa.

TEMPORINI ER; KARA-JOSÉ N; GONDIM EL \& DANTAS FJ. Knowledge on ocular health among university hospital staff Medicina, Ribeirão Preto, 35: 53-61, jan./march 2002.

ABSTRACT: Design of the study: Observational/exploratory survey. Objective: To verify knowledge on ocular health among university hospital staff. Methodology: An open questionnaire on ocular diseases (cataract, glaucoma, diabetic retinopathy and procedures on chemical burn) was applied to health personnel. Results: The sample was composed by 122 individuals: 30 senior nurses (24.6\%), 52 nursing assistants (42.6\%), 24 administrative workers (19.7\%), and 16 employees working in the ophthalmologic clinic (13.1\%). They have shown knowledge on what caratact means $(83.3 \%$ of the nurses and $75.0 \%$ of the ophthalmology staff). Among groups who belong to other areas differentfrom ophthalmology, was misunderstood the real concept of cataract . Glaucoma is unknown by $79.1 \%$ of the hospital's administrative employees; $37.6 \%$ of the ophthalmology service personnel declare having no knowledge concerning cure for, or control over, this vision affection. The non-acquaintance with the consequences of diabetes for the vision system was observed in all groups. Conclusion: The lack of knowledge on ocular health was observed in all groups. The development of training programs for the hospital staff with the purpose of multiplying eye health education to the hospital patients and in the social and cultural environment is suggested.

UNITERMS: Eye Health. Health Education. Patient Care Team. Blindness; prevention. 


\section{REFERÊNCIAS BIBLIOGRÁFICA}

1 - GREEN LW \& KREUTER M W. Health promotion planning an educational and environmental approach. $2^{\text {nd }} \mathrm{ed}$. Mountain View, Mayfield, p. 154-173, 1991.

2 - TEMPORINI ER \& KARA-JOSÉ N . Níveis de prevenção de problemas oftalmológicos: propostas de investigação. Arq Bras Oftalmol 58:189-192, 1995.

3 - CANDEIAS NMF. The concepts of health education and promotion - individual and organizational changes. Rev Saúde Pública 31: 209-213, 1997.

4 - BRICEÑO-LEON R . Siete tesis sobre la educación sanitária para la participación comunitária. Cad Saúde Pública 12: 730, 1996.

5 -WORLD HEALTH ORGANIZATION. Strategies for the prevention of blindness in national programmes- a primary health care approach. WHO, Geneva,1984.

6 - RICE MA framework for developing health promotion and education iniciatives in reproductive health. Promot Educ 3: 710, 1996.

7 - ROCHA JCP; GONDIM EL; BRAGA FTC ; DANTAS FJ ; TEMPORINI ER \& KARA-JOSÉ N . Ocular health myths among a hospital staff. Ophthalmic Epidemiol 4: 107-113, 1997.

8 - VERDAGUER T J. One world, one voice, one vision. Arq Bras Oftalmol 61:5-6, 1998.

9 - ELLWEIN L B \& KUPFER C . Strategic issues in preventing cataract blindnessin developing countries. Bull World Health Organ 73: 681-690, 1995.
10 - KARA-JOSÉ N; CONTRERAS F; CAMPOS MA; DELGADO AM; MOWERY RL \& ELLWEIN L B . Screening and surgical intervention results from cataract- free zones projects in Campinas, Brazil and Chimbote, Peru. Int Ophthalmol 14: 155-164, 1990.

11 - TEMPORINI E R; KARA-JOSÉ N \& KARA-JOSÉ JR. N . Catarata senil: características e percepções de pacientes atendidos em projeto comunitário de reabilitação visual. Arq Bras Oftalmol 60: 79-83, 1997.

12 - SOMMER A . Organizing to prevent third world blindness. Am J Ophthalmol 107: 544-546, 1989.

13 - KARA-JOSÉ N \& TEMPORINI ER. Cirurgia da catarata: o porquê dos excluídos. Rev Panamer Saúde Pública 6: 242-248., 1999.

14 - GULLO RM; COSTA VP; BERNARDI L \& KARA-JOSÉ N. Condições visuais de pacientes glaucomatosos em um hospital universitário. Arq Bras Oftalmol 59: 147-150, 1996.

15 - THYLEFORS B; NÉGREL AD; PARARAJASEGARAM R \& DADZIE KY. Global data on blindness. Bull World Health Organ 73: 115-121, 1995.

16 - BOWER NK. A primary care team approach to the prevention of ocular complications of diabetes: a program review. J Am Optom Assoc 68: 233-242, 1997.

Recebido para publicação em 19/11/2001

Aprovado para publicação em 25/02/2002 


\section{ANEXO \\ Questionário não estruturado}

1. Você sabe o que é catarata? Como você a define?

2. Existe tratamento para catarata?

3. Você sabe o que é glaucoma? Existe cura para o glaucoma?

4. Pode haver conseqüência do diabete para os olhos? Quais?

5. Em casa, quando acontece algum acidente com os olhos, deve-se fazer algo ou não? O que fazer? 